\title{
Plasmon molecules in overlapping nanovoids
}

\author{
I. Romero, ${ }^{1}$ T. V. Teperik, ${ }^{1,2}$ and F. J. García de Abajo ${ }^{1,3, *}$ \\ ${ }^{1}$ Donostia International Physics Center, Apartado 1072, 20080 San Sebastian, Spain \\ ${ }^{2}$ Institute of Radio Engineering and Electronics (Saratov Division), Russian Academy of Sciences, Zelyonaya 38, 410019 Saratov, Russia \\ ${ }^{3}$ Instituto de Óptica-CSIC, Serrano 121, 20006 Madrid, Spain
}

(Received 11 January 2008; published 7 March 2008)

\begin{abstract}
Coupled and shape-tailored metallic nanoparticles are known to exhibit hybridized plasmon resonances. This paper discusses the optical properties of a complementary system formed by overlapped nanovoid dimers buried in gold and filled with silica. This is an alternative route for plasmon engineering that benefits from vanishing radiation losses. Our analysis demonstrates the possibility of designing artificial plasmon molecules on the basis of void plasmon hybridization, which allows fine mode tuning by varying the overlap between voids. The proposed structures could find application to both signal processing through buried optical elements and tunable-plasmon biosensing.
\end{abstract}

DOI: 10.1103/PhysRevB.77.125403

PACS number(s): 73.20.Mf, 78.67.-n, 84.40.Az

\section{INTRODUCTION}

Plasmons in metallic particles have recently attracted considerable attention as promising candidates to realize photonic devices, enabling manipulation of light over submicron distances. The frequency of plasmon resonances can be tuned at will by nanoengineering the particle environment and playing with different geometries. ${ }^{1-8}$ The suite of available nontrivial structures includes nanoshells, ${ }^{1,2}$ nanorings, ${ }^{3}$ nanovoids, ${ }^{4}$ nanorods, ${ }^{6}$ and exotic polyhedral crystallites. ${ }^{8}$ Plasmon hybridization originating in intraparticle ${ }^{1}$ and interparticle ${ }^{5}$ interactions adds up an extra handle to finetuning resonant mode energies, particularly in dimers near the touching limit. ${ }^{7,9}$

In this context, the guiding properties of nanoparticle linear arrays have been thoroughly investigated with a view to designing optical interconnects based upon plasmon hopping between particles. ${ }^{10,11}$ However, plasmon propagation in these systems is found to be severely damaged by both dissipation in the metal and radiative losses. The latter are minimized by relying on small particles $(\leqslant 50 \mathrm{~nm})$, but there is a trade-off between both types of losses. Ultimately, absorption limits propagation to submicron distances in small particles. ${ }^{11}$ This problem can be solved by using instead larger voids or dielectric inclusions buried in metal. Actually, the radiative damping of hybridized voidlike plasmons in nanoshells decreases extremely rapidly with increasing shell thickness. ${ }^{2}$ The basic unit of such void arrays is the void dimer, to which we devote this work.

In the electrostatic limit, the mode frequencies of the particle array, which we can denote $\omega_{j}^{p}$ (here, $j$ runs over different modes and the superscript $p$ refers to particle), and its complementary void array $\omega_{j}^{v}$ are interconnected through the general, exact relation $\left(\epsilon_{i}+\epsilon_{o}\right) /\left(\epsilon_{i}-\epsilon_{o}\right)=\Lambda_{j},{ }^{12}$ where $\epsilon_{i}$ and $\epsilon_{o}$ are the permittivities of the material inside and outside the spherical boundaries, respectively, and $\Lambda_{j}$ are eigenvalues that depend exclusively on geometry and not on the actual dielectric properties of the materials involved. When the media under consideration are air and a Drude metal of bulk plasma frequency $\omega_{p}$ and damping parameter $\eta$ described by

$$
\epsilon(\omega)=1-\frac{\omega_{p}^{2}}{\omega(\omega+i \eta)},
$$

one obtains the sum rule $\left(\omega_{j}^{p}\right)^{2}+\left(\omega_{j}^{v}\right)^{2}=\omega_{p}^{2}{ }^{13}$ Therefore, the analysis of electrostatic void arrays is fully contained in the electrostatic particle array. We will, however, consider relatively large voids yielding longer propagation distances. Then, retardation effects become dominant for sizes above the skin depth $(\sim 25 \mathrm{~nm}$ at visible and NIR frequencies), and therefore a separate analysis becomes necessary.

In this work, we describe the optical properties of plasmon molecules formed in overlapping nanovoids buried in metal. Similar to particle dimers, ${ }^{5}$ large shifts of hybridized modes are observed, controlled by varying the degree of overlap, while significant field enhancement takes place near the junction region. However, unlike particle dimers, void dimers do not present a singular transition near touching conditions. More precisely, the unphysical mode observed in the separate particle dimer, ${ }^{7}$ characterized by a net induced charge sitting in each particle, becomes now physical for the voids, although the intervoid interaction is drastically reduced in nonoverlapping systems due to the skin-depth effect.

\section{PLASMON HYBRIDIZATION IN OVERLAPPED NANOVOIDS}

We rigorously solve Maxwell's equations in the frequency domain $\omega$ for overlapping void dimers filled with silica using the boundary element method (BEM), ${ }^{14,15}$ in which surface charges and currents are introduced to match the fields across dielectric boundaries, thus resulting in a set of surfaceintegral equations that is transformed in turn into a linearalgebra problem upon discretization of the integrals. The NIR permittivity of gold is modeled for simplicity through Eq. (1) with parameters $\omega_{p}=7.9 \mathrm{eV}$ and $\eta=0.09 \mathrm{eV},{ }^{16}$ while we take $\epsilon_{i}=2$ for silica.

Fully buried voids are unaccessible to external radiation, so light scattering cannot be employed to characterize them. Instead, we investigate the photonic local density of states (LDOS) in the silica region. Similar to its electron counter- 
part in solid state physics, the LDOS is defined as the combined local intensity of all eigenmodes of the system under consideration. ${ }^{17}$ With this definition, the LDOS $\rho$ projected along the direction of an atomic-decay transition dipole $\mathbf{D}$ is related to the decay rate as $\Gamma=\left(4 \pi^{2} \omega D^{2} / \hbar\right) \rho .^{17,18}$ This is, in fact, the electric part of the LDOS, which is the one relevant in plasmonic structures like in our study. A complete definition of the LDOS should include, however, a magnetic part as well. ${ }^{19}$ In practical terms, the LDOS can be calculated from the field scattered by the void boundaries, projected along the dipole, according to ${ }^{20}$

$$
\rho=\frac{\omega^{2} \sqrt{\epsilon_{i}}}{3 \pi^{2} c^{3}}+\frac{1}{2 \pi^{2} \omega D^{2}} \operatorname{Im}\left\{\mathbf{D}^{*} \cdot \mathbf{E}^{\text {scat }}\right\}
$$

where the first term on the right hand side is the silica LDOS. Here, we consider projection directions parallel and perpendicular to the dimer axis and give the LDOS normalized to its value in silica.

We show in Fig. 1 the LDOS at the center of one of the silica-filled voids of a dimer formed by spheres of radius $a=240 \mathrm{~nm}$ with $d=60 \mathrm{~nm}$ overlap (solid curves). For comparison, the isolated-void LDOS is also represented (dashed curves), exhibiting a prominent feature corresponding to the $l=1$ electric Mie resonance in agreement with the analytical expression (see arrows) ${ }^{21}$

$$
\epsilon_{o} h_{l}^{(1)}\left(\rho_{o}\right) \frac{d\left[\rho_{i} j_{l}\left(\rho_{i}\right)\right]}{d \rho_{i}}=\epsilon_{i} j_{l}\left(\rho_{i}\right) \frac{d\left[\rho_{o} h_{l}^{(1)}\left(\rho_{o}\right)\right]}{d \rho_{o}},
$$

where $\rho_{j}=\sqrt{\epsilon_{j}} \omega a / c, j_{l}$ and $h_{l}^{(1)}$ are spherical Bessel and Hankel functions, and $l$ is the orbital momentum number. The small mismatch between BEM calculations and the analytical mode frequency originates in nonresonant contributions to the dipole-induced field of the former.

The $l$-plasmon mode of the single void is $2 l+1$ degenerate, and one can expect partial lifting of this degeneracy due to void overlap. This is clearly observed in Fig. 1. The resulting modes have $m=0$ and $m= \pm 1$ azimuthal symmetry in Figs. 1(a) and 1(b), corresponding to transition dipoles oriented parallel and perpendicular to the dimer axis, respectively. The latter is doubly degenerate, so that the overall number of six modes is preserved after intervoid interaction is switched on. The dimer modes are hybridized and energy split, but the splitting is significantly larger for the transverse low-energy feature D. The origin of this can be traced back to strong interaction within the intervoid opening, which is made possible for codirectional transverse orientations of the exciting dipoles and has similar nature as gap modes in particle dimers. ${ }^{5}$ In a related context, this gives rise to rim modes localized at the opening of voids partly buried in flat metal surfaces. ${ }^{22}$

This intuition is supported by the induced-field distributions shown in Fig. 2 for features A-E of Fig. 1. See, for instance, the large increase in field intensity near the void junction region associated to peak D. This is accompanied by significant piling up of induced charge, as shown in the graph to the right of the contour plot. However, peak E has vanishing intensity in the void opening due its quadrupolar symmetry, and therefore it suffers a rather moderate shift.
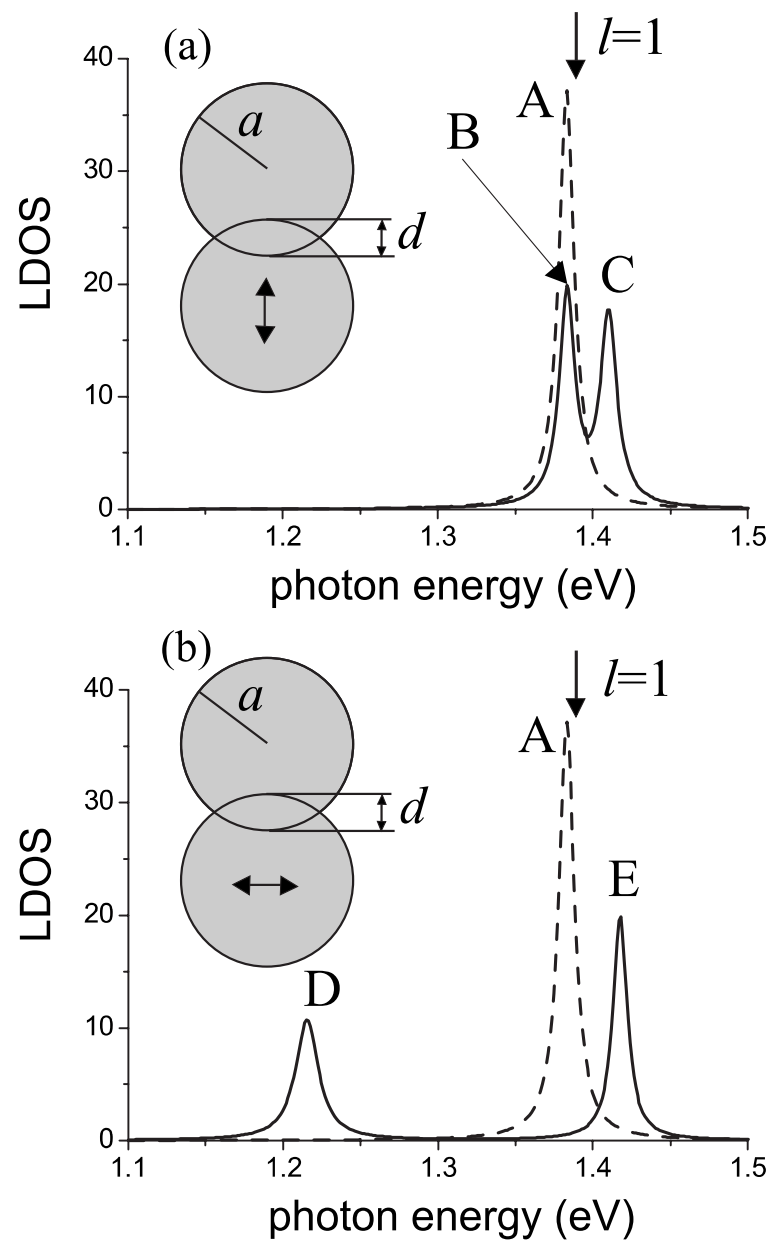

FIG. 1. Plasmon modes in overlapping nanovoids. The photon local density of states (LDOS) is calculated inside two overlapping silica inclusions in gold (solid curves) and projected along directions (a) parallel and (b) perpendicular to the dimer axis (see insets). The inclusions are spheres of radius $a=240 \mathrm{~nm}$. The overlapping distance is $d=60 \mathrm{~nm}$. The LDOS of a single inclusion is shown as dashed curves. The vertical arrows mark the dipole plasmon energy of a single inclusion obtained from Eq. (2) with $l=1$.

These results suggest the possibility to design plasmon molecules of tailored frequency and symmetry. For example, a quadrupole plasmon mode can be obtained in the dimer near the single-void dipole mode frequency (E in Figs. 1 and 2). More sophisticated plasmon modes should also be possible by combining several voids, with the dimer acting as a building block.

In contrast to metallic-particle dimers, in which plasmons interact through a dielectric environment, ${ }^{7}$ our void plasmons are coupled only through the region of overlap. Therefore, plasmon mixing critically depends on the overlap distance $d$ and can be significantly reduced in buried geometries, thus simplifying the understanding of the resulting plasmon molecule. In particular, modes B, C, and E are reliably described in terms of dipole-dipole interaction, whereas mode $\mathrm{D}$, which lies further away from the single-void dipole resonance, is dominated by the rimlike mode, involving a large degree of mixing of higher-order modes. 


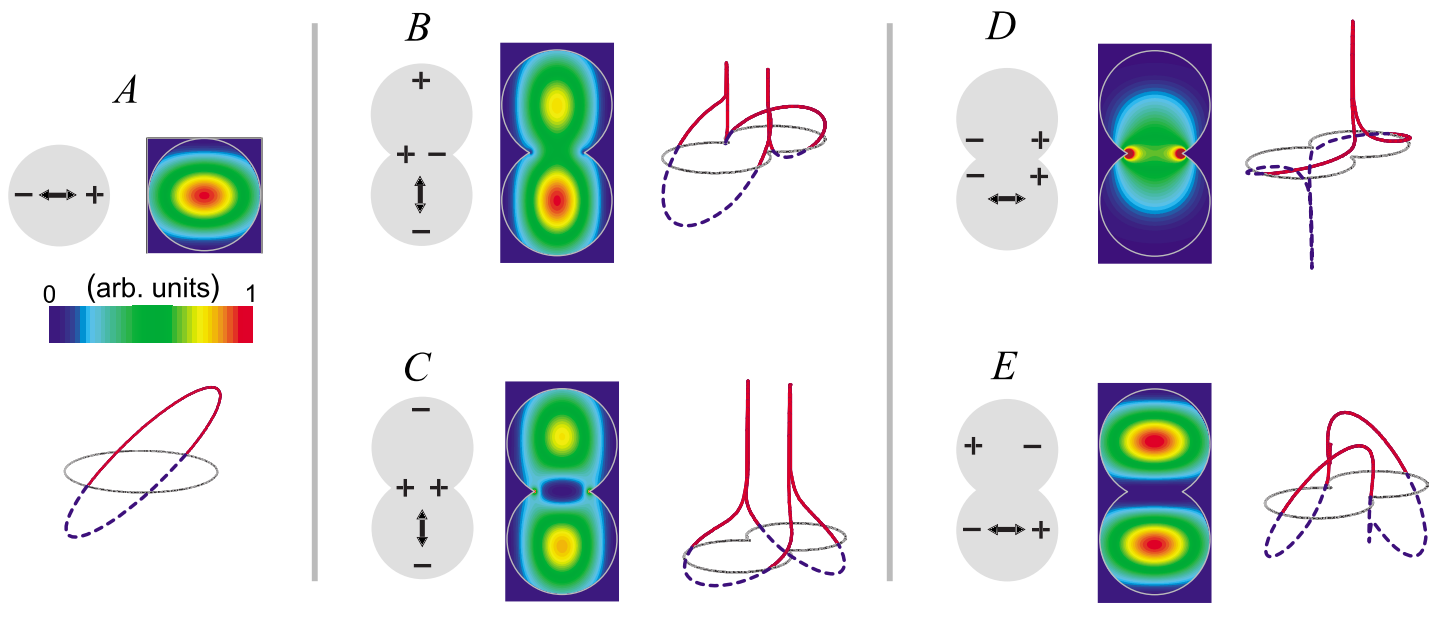

FIG. 2. (Color online) Plasmon mode maps corresponding to peaks A-E in Fig. 1. The contour plots show the square of the induced electric field produced by dipoles oriented as indicated by the double arrows. Schemes of the charge distribution are shown to the left of each contour plot. The induced surface charge is shown as well, with negative and positive values represented in blue (dashed) and red (solid), respectively.

\section{EVOLUTION OF BURIED-DIMER VOIDS WITH OVERLAP DISTANCE}

The full $d$ dependence of the void dimers is presented in Fig. 3. Several characteristic features are clearly resolved in the evolution of the plasmon modes.

(i) Mode splitting is consistently larger for the transverse orientation of the dipole, in good agreement with the conclusions extracted from Figs. 1 and 2.

(ii) As anticipated above, the interaction between voids switches on smoothly between nontouching and overlapping configurations, in contrast to the singular transition observed for particle dimers. ${ }^{7}$

(iii) The low-energy dipole modes evolve smoothly toward the single-void dipole when the overlap is complete $(d=2 a)$. An initial redshift after touching is followed by a blueshift as $d$ increases. In the rimlike mode [see D in Figs. 1 and 2, and low-frequency features in Fig. 3(b)], a maximum redshift is produced at an optimum distance resulting from the compromise between increasing mode volume as $d$ becomes smaller (this lowers the kinetic energy of the mode) and increasing intervoid interaction as $d$ increases (this lowers the mode energy due to intermode attraction).

(iv) The high-energy quadrupolar modes march toward the single-void quadrupole as $d$ increases and the quadrupole-mode LDOS becomes zero at the center of a single void.

(v) A weak transition resonance is observed in the vicinity of $1.7 \mathrm{eV}$ [Fig. 3(b)], originating in higher-order void multipoles that are the counterpart of the leftmost rimlike mode.

The plasmon widths determine how well the modes are defined. In this sense, the $1.7 \mathrm{eV}$ resonance just noted is a well-defined one. In fact, the widths of all our reported plasmons arise from metal absorption, so that modes associated with fields mainly contained in the silica and characterized by minimum overlap with the metal will have longer lifetimes. This is clear when comparing modes D and E in Figs. 1(b) and 2: D is broader because it has larger intensity in the
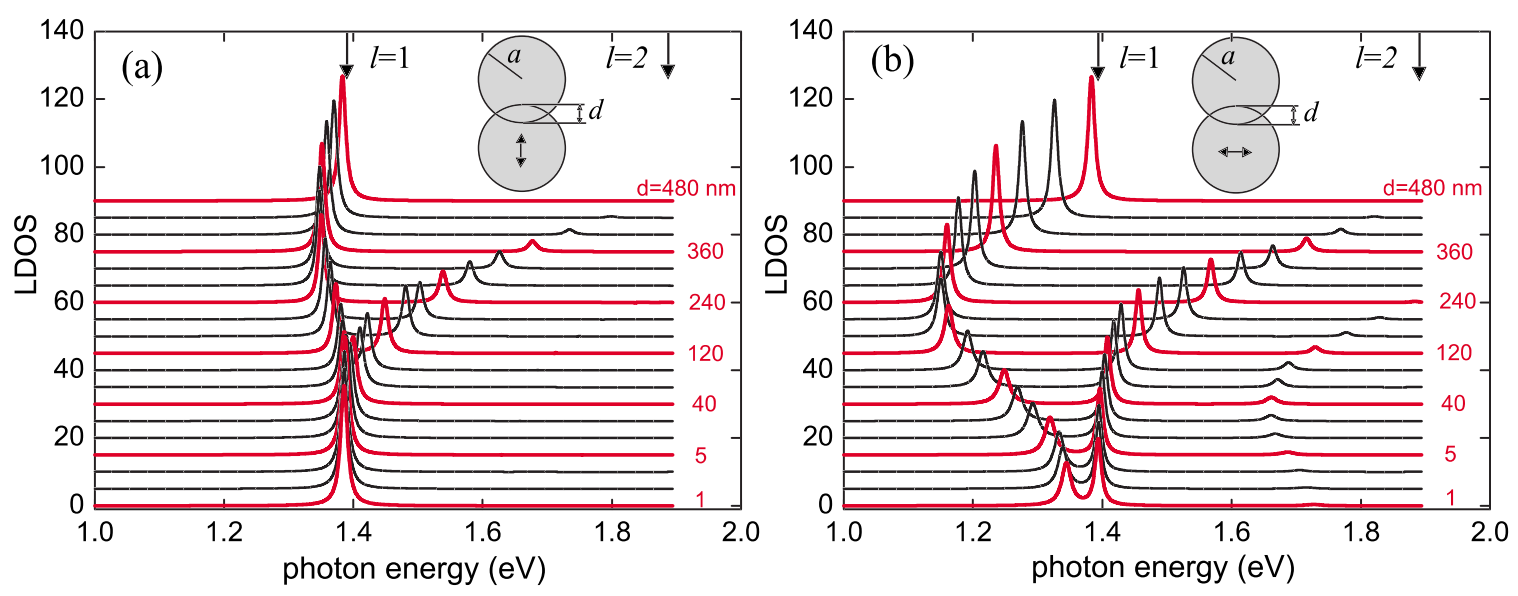

FIG. 3. (Color online) Evolution of void-dimer plasmon energies with overlapping distance $d$. The modes are probed through the LDOS at the center of one of the voids, projected along the dimer axis in (a) and perpendicular to that axis in (b). The void radius is $a=240 \mathrm{~nm}$. The vertical arrows mark the energy of the dipole $(l=1)$ and quadrupole $(l=2)$ plasmon modes of a single inclusion obtained from Eq. $(2)$. 


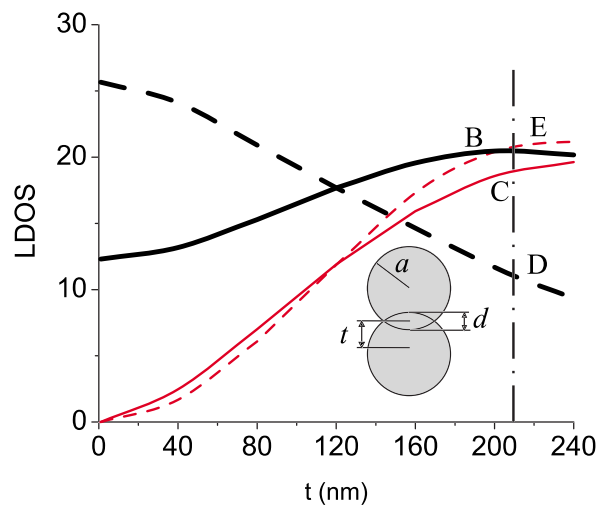

FIG. 4. (Color online) LDOS calculated for two overlapping silica inclusions in gold as a function of position $t$ along the dimer axis. The LDOS is projected along parallel (solid curves) and perpendicular (dashed curves) directions relative to that axis. Black (thick) and red (thin) curves correspond to low- and high-energy resonances, respectively. The void radius is $a=240 \mathrm{~nm}$ and the overlapping distance is $d=60 \mathrm{~nm}$.

metal (see region near the void junction). However, much of the field intensity lies in the silica, and that is why the mode widths are considerably smaller than $\eta$ in Eq. (1).

Finally, the location of the probing dipole determines the actual degree of coupling to the plasmons. This is illustrated in Fig. 4, which shows the evolution of the plasmon LDOS along the dimer axis for features B-E of Fig. 1. Interestingly, only the low-energy hybrid plasmons (B and D), which have the original dipole symmetry, contribute to the LDOS at the dimer center (thick curves). The high-energy modes have an overall quadrupolar character and can only be excited by dipoles displaced with respect to the dimer central region.

\section{CONCLUSIONS}

We have investigated the optical properties of two overlapping silica inclusions embedded in gold and found hybridized plasmon modes, the mixing of which can be effectively controlled by changing the degree of geometrical overlap. These structures open up the possibility to realize radiativefree plasmon molecules through dielectric inclusions buried in metal. Large field enhancements are observed on resonance near the overlap region, which can find application to surface-enhanced Raman spectroscopy and other forms of biosensing.

The void dimer constitutes the basic building block of buried optical circuits for future on-chip interconnects and switching elements. In contrast to particle arrays, void arrays will benefit from minimum cross talk across the metal and vanishing radiative losses, thus allowing a step forward in nanoscale integration.

\section{ACKNOWLEDGMENTS}

This work has been supported in part by the Spanish MEC (NAN2004-08843-C05-05, MAT2007-66050, and consolider NanoLight.es) and by the EU-FP6 (NMP4-2006-016881 "SPANS"). T.V.T. acknowledges support from the Russian Academy of Sciences and Russian Foundation for Basic Research (Grant Nos. 05-02-17513, 07-02-91011, and 06-0281007)
*Author to whom correspondence should be addressed: jga@cfmac.csic.es

${ }^{1}$ E. Prodan, C. Radloff, N. J. Halas, and P. Nordlander, Science 302, 419 (2003)

${ }^{2}$ T. V. Teperik, V. V. Popov, and F. J. García de Abajo, Phys. Rev. B 69, 155402 (2004).

${ }^{3}$ J. Aizpurua, P. Hanarp, D. S. Sutherland, M. Käll, G. W. Bryant, and F. J. García de Abajo, Phys. Rev. Lett. 90, 057401 (2003).

${ }^{4}$ T. V. Teperik, V. V. Popov, and F. J. García de Abajo, Phys. Rev. B 71, 085408 (2005).

${ }^{5}$ P. Nordlander, C. Oubre, E. Prodan, K. Li, and M. I. Stockman, Nano Lett. 4, 899 (2004).

${ }^{6}$ C. Burda, X. Chen, R. Narayanan, and M. A. El-Sayed, Chem. Rev. (Washington, D.C.) 105, 1025 (2005).

${ }^{7}$ I. Romero, J. Aizpurua, G. W. Bryant, and F. J. García de Abajo, Opt. Express 14, 9988 (2006).

${ }^{8}$ A. Sánchez-Iglesias, I. Pastoriza-Santos, J. Pérez-Juste, B. Rodríguez-González, F. J. García de Abajo, and L. Liz-Marzán, Adv. Mater. (Weinheim, Ger.) 18, 2529 (2006).

${ }^{9}$ M. Danckwerts and L. Novotny, Phys. Rev. Lett. 98, 026104 (2007).

${ }^{10}$ J. C. Weeber, J. R. Krenn, A. Dereux, B. Lamprecht, Y. Lacroute, and J. P. Goudonnet, Phys. Rev. B 64, 045411 (2001).
${ }^{11}$ S. A. Maier, P. G. Kik, H. A. Atwater, S. Meltzer, E. Harel, B. E. Koel, and A. A. G. Requicha, Nat. Mater. 2, 229 (2003).

${ }^{12}$ F. J. García de Abajo and J. Aizpurua, Phys. Rev. B 56, 15873 (1997).

${ }^{13}$ S. P. Apell, P. M. Echenique, and R. H. Ritchie, Ultramicroscopy 65, 53 (1996).

${ }^{14}$ F. J. García de Abajo and A. Howie, Phys. Rev. Lett. 80, 5180 (1998).

${ }^{15}$ F. J. García de Abajo and A. Howie, Phys. Rev. B 65, 115418 (2002).

${ }^{16}$ M. Kreiter, S. Mittler, W. Knoll, and J. R. Sambles, Phys. Rev. B 65, 125415 (2002).

${ }^{17}$ D. P. Fussell, R. C. McPhedran, and C. Martijn de Sterke, Phys. Rev. E 70, 066608 (2004).

${ }^{18}$ S. M. Barnett and R. Loudon, Phys. Rev. Lett. 77, 2444 (1996).

${ }^{19}$ K. Joulain, R. Carminati, J. P. Mulet, and J. J. Greffet, Phys. Rev. B 68, 245405 (2003).

${ }^{20}$ L. A. Blanco and F. J. García de Abajo, Phys. Rev. B 69, 205414 (2004).

${ }^{21}$ L. A. Weinstein, Open Resonators and Open Waveguides (Golem, New York, 1969).

${ }^{22}$ R. M. Cole, J. J. Baumberg, F. J. García de Abajo, S. Mahajan, M. Abdelsalam, and P. N. Bartlett, Nano Lett. 7, 2094 (2007). 